\title{
MINKOWSKI'S INEQUALITY BASED SENSITIVITY ANALYSIS OF FUZZY SIGNATURES
}

\author{
István Á. Harmati ${ }^{1}$, Ádám Bukovics ${ }^{2}$, and László T. Kóczy ${ }^{3}$ \\ ${ }^{1}$ Department of Mathematics and Computational Sciences \\ Széchenyi István University, Egyetem tér 1, 9026 Györ, Hungary \\ harmati@sze.hu \\ ${ }^{2}$ Department of Structural and Geotechnical Engineering \\ Széchenyi István University, Egyetem tér 1, 9026 Gyór, Hungary \\ ${ }^{3}$ Department of Automation \\ Széchenyi István University, Egyetem tér 1, 9026 Györ, Hungary \\ Department of Telecommunications and Media Informatics \\ Budapest University of Technology and Economics, Budapest, Hungary
}

\begin{abstract}
Fuzzy signatures were introduced as special tools to describe and handle complex systems without their detailed mathematical models. The input parameters of these systems naturally have uncertainties, due to human activities or lack of precise data. These uncertainties influence the final conclusion or decision about the system. In this paper we discuss the sensitivity of the weigthed general mean aggregation operator to the uncertainty of the input values, then we analyse the sensitivity of fuzzy signatures equipped with these aggregation operators. Finally, we apply our results to a fuzzy signature used in civil enginnering.
\end{abstract}

Keywords: aggregation operators, generalized mean, sensitivity analysis, fuzzy signatures, building diagnostics

\section{Introduction}

The problem of modelling and classification of complex objects and systems often arises in many fields of science and technology. Frequent difficulty is that there appear not well-known or hidden interdependencies between the variables. Moreover, in many cases there are not known accurate mathematical models, and because of lack of reproducibility the statistical tools can be used with large limitations to check the assumptions about the system.

Fuzzy signatures are possible tools to describe such complex systems and objects. In this kind of approach, complex systems are described by a set of qualitative measures, which are also arranged into a hierarchical framework expressing interconnections and dependencies, and modelling the human approach to the problem.

The fuzzy signature based modelling technique can be applied for very different problems, for example in economy, in the medical field [1], and in several fields of engineering and informatics, for example robotics [2], data mining [3] and civil engineering [4].

In a mathematical point of view, fuzzy signatures are hierarchical representations of data structuring into vectors of fuzzy values [5]. A fuzzy signature is defined as a special multidimensional fuzzy data structure, which is a generalization of 
vector valued fuzzy sets [6]. Vector valued fuzzy sets are special cases of $L$-fuzzy sets which were introduced in [7]. A fuzzy signature is defined by

$$
A: X \rightarrow S^{(n)},
$$

where $X$ is the universe of discourse, $1 \leq n$ and

$$
S^{(n)}=\times_{i=1}^{n} S_{i}, \quad S_{i}=\left\{\begin{array}{l}
{[0,1]} \\
S^{(m)} .
\end{array}\right.
$$

We can represent a fuzzy signature by nested vector value fuzzy sets and also by a tree graph (see Figure 1 ), which is much more understandable [6].

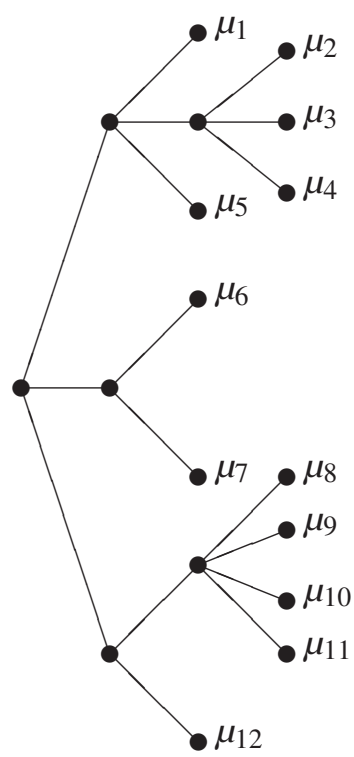

Figure 1. A fuzzy signature graph and the corresponding nested vectors.

The input values $(\mu-\mathrm{s})$ of the fuzzy signature usually given by human experts or estimation methods. The final output (at the root of the tree graph) is computed from the inputs applying suitable aggregation functions, this is the membership value of the whole fuzzy signature. Since different human experts could assign slightly different values to the same situation, a usable fuzzy signature based model should not be too sensitive to the input values.

The goal of this article is to discuss how the membership value of the whole fuzzy set changes if the membership values in the nested vectors change. In other words, if we think of the tree graph representation, how the membership value of the root changes if the membership values of leaves change. To answer this question we have to know how to compute a membership value of a subgraph from the leaves.

In this article we assume that all the operators applied on membership values in the signature are from the class of weighted generalized mean aggregation operators (WGMs). A similar question was investigated in [8], but for different cases and by different mathematical tools.

There are several approaches discussing the sensitivity of aggregation operators with different weights, for example [9] discusses the sensitivity of weighted fuzzy aggregation, [10] and [11] discuss the sensitivity of ordered weighted aggregation operators.

The paper is organized as follows: in Section 2 we recall some mathematical tools, in Section 3 the sensitivity of WGM is discussed, in Section 4 we examine the sensitivity of fuzzy signatures in general and in special cases, and finally in Section 5 we discuss the sensitivity of a concrete fuzzy signature used in civil engineering.

\section{Mathematical Background}

The generalized mean and its generalization, the weighted generalized mean form a very large class of aggregation operators. Their various special cases often arise also in theoretical and practical problems.

Definition 1 (Generalized mean) (see for example [12] or [13]) Let $x_{1}, \ldots, x_{n}$ be nonnegative real numbers and $p \in \mathbb{R}(p \neq 0)$. Then their generalized mean with parameter $p$ :

$$
M_{p}\left(x_{1}, \ldots, x_{n}\right)=\left[\frac{1}{n} \sum_{k=1}^{n} x_{k}^{p}\right]^{\frac{1}{p}}
$$

Some special cases in $p$ :

$-p=1 \quad$ arithmetic mean

$-p=2$ quadratic mean

$-p=-1$ harmonic mean 
Definition 2 (Weighted generalized mean; WGM) Let $x_{1}, \ldots, x_{n}$ and $w_{1}, \ldots, w_{n}$ be nonnegative real numbers, $w_{i} \geq 0, \sum_{i=1}^{n} w_{i}=1$ and $p \in \mathbb{R}(p \neq 0)$.

Then the weighted generalized mean of $x_{1}, \ldots, x_{n}$ with weights $w_{1}, \ldots, w_{n}$ and with parameter $p$ :

$$
M_{p}^{w}\left(x_{1}, \ldots, x_{n}\right)=\left[\sum_{k=1}^{n} w_{k} x_{k}^{p}\right]^{\frac{1}{p}} .
$$

We note here that the weighted generalized mean sometimes refered as 'scaled norm'. We do not use this terminology because of the possible missunderstanding: the properties of the norm are fulfilled only when $p \geq 1$, but the WGM is defined for every $p \in \mathbb{R}$.

The generalized mean is a special case of the weighted generalized mean with weights $w_{k}=\frac{1}{n}$. The limits at $\pm \infty$ regardless to the weight:

$$
\begin{aligned}
& \lim _{p \rightarrow \infty}\left[\sum_{k=1}^{n} w_{k} x_{k}^{p}\right]^{\frac{1}{p}}=\max \left(x_{i}\right), \\
& \lim _{p \rightarrow-\infty}\left[\sum_{k=1}^{n} w_{k} x_{k}^{p}\right]^{\frac{1}{p}}=\min \left(x_{i}\right) .
\end{aligned}
$$

The limit if $p \rightarrow 0$ is the weighted geometric mean:

$$
\lim _{p \rightarrow 0}\left[\sum_{k=1}^{n} w_{k} x_{k}^{p}\right]^{\frac{1}{p}}=\prod_{i=1}^{n} x_{i}^{w_{i}} .
$$

Our aim is to give upper bound on the change of the weighted generalized mean if we know the change of the input values $x_{1}, \ldots, x_{n}$. In Section 3 we search for such a bound for $|\Delta M|$ which depends on $\underline{\Delta x}$ or on a kind of vector norm of $\Delta \underline{x}$, and on the basis of these results we give upper bounds for the change of the whole fuzzy signature in Section 4.

First we recall the definition of the $p$-norm (see for example [14]).

Definition 3 (p-norm) Let $p \geq 1$ a real number and $\underline{x}=\left(x_{1}, \ldots, x_{n}\right) \in \mathbb{R}^{n}$. Then the $p$-norm of $\underline{x}$

$$
\|\underline{x}\|_{p}=\left(\sum_{k=1}^{n}\left|x_{k}\right|^{p}\right)^{\frac{1}{p}} .
$$

Some widely used $p$-norms:
- $p=1$ (taxicab norm) $\|\underline{x}\|_{1}=\left|x_{1}\right|+\ldots+\left|x_{n}\right|$,

- $p=2$ (euclidean norm) $\|\underline{x}\|_{2}=\sqrt{x_{1}^{2}+\ldots+x_{n}^{2}}$,

$-p=\infty$ (maximum norm),

$\|\underline{x}\|_{\infty}=\max \left(\left|x_{1}\right|, \ldots,\left|x_{n}\right|\right)$.

Two important properties of the $p$-norm:

- If $1 \leq p \leq q \leq \infty$ then $\|\underline{x}\|_{q} \leq\|\underline{x}\|_{p}$,

- If $1 \leq p \leq q \leq \infty$ then $\|\underline{x}\|_{p} \leq\|\underline{x}\|_{q} \cdot n^{1 / p-1 / q}$.

We will use the generalization of the triangular inequality, the so called Minkowski's inequality.

Theorem 1 (Minkowski's inequality) (see for example [12] or [13]) Let $\underline{a}, \underline{b} \in \mathbb{R}^{n}, p \geq 1$, then the following inequality holds:

$$
\|\underline{a}+\underline{b}\|_{p} \leq\|\underline{a}\|_{p}+\|\underline{b}\|_{p} .
$$

The generalization of the reverse triangular inequalty also holds:

Corollary 1 If $\underline{a}, \underline{b} \in \mathbb{R}^{n}, p \geq 1$, then,

$$
\left|\|\underline{a}\|_{p}-\|\underline{b}\|_{p}\right| \leq\|\underline{a}-\underline{b}\|_{p} .
$$

\section{Sensitivity of the Weighted Gen- eral Mean for $p \geq 1$}

In this Section we analyse the change of the WGM under the change of its input vector. Note that we examine the case $p \geq 1$. Let us use the following notations:

$$
\begin{aligned}
\underline{w}^{1 / p} & =\left(w_{1}^{1 / p}, \ldots, w_{n}^{1 / p}\right), \\
\underline{w}^{1 / p} \cdot \underline{x} & =\left(w_{1}^{1 / p} \cdot x_{1}, \ldots, w_{n}^{1 / p} \cdot x_{n}\right) .
\end{aligned}
$$

If the input vector is $\underline{x}=\left(x_{1}, \ldots, x_{n}\right)$, the vector if the weights is $\underline{w}=\left(w_{1}, \ldots, w_{n}\right)$, then the weighted generalized mean with parameter $p$ is

$$
\begin{aligned}
M & =\left[\sum_{i=1}^{n} w_{i} x_{i}^{p}\right]^{\frac{1}{p}}=\left[\sum_{i=1}^{n}\left(w_{i}^{1 / p} x_{i}\right)^{p}\right]^{\frac{1}{p}}, \\
& =\left\|\underline{w}^{1 / p} \cdot \underline{x}\right\|_{p}
\end{aligned}
$$

If the new (maybe perturbed) input vector is $\underline{x}^{*}=\left(x_{1}^{*}, \ldots, x_{n}^{*}\right)$, then the new output is $M^{*}=$ $\left\|\underline{w}^{1 / p} \cdot \underline{x}^{*}\right\|_{p}$. So the change of the input is $\Delta \underline{x}=$ 
$\underline{x}^{*}-\underline{x}$, the change of the output is $\Delta M=M^{*}-M$. In the following we give upper estimations for $|\Delta M|$.

$$
\begin{aligned}
|\Delta M| & =\left|\left\|\underline{w}^{1 / p} \cdot \underline{x}^{*}\right\|\right|_{p}-\left\|\underline{w}^{1 / p} \cdot \underline{x}\right\|_{p} \mid \\
& \leq\left\|\underline{w}^{1 / p} \cdot \underline{x}^{*}-\underline{w}^{1 / p} \cdot \underline{x}\right\|_{p}, \\
& =\left\|\underline{w}^{1 / p} \cdot\left(\underline{x}^{*}-\underline{x}\right)\right\|_{p}=\left\|\underline{w}^{1 / p} \cdot \Delta \underline{x}\right\|_{p}, \\
& =\left[\sum_{i=1}^{n}\left(w_{i}^{1 / p}\left|\Delta x_{i}\right|\right)^{p}\right]^{\frac{1}{p}}=\left[\sum_{i=1}^{n} w_{i} \cdot\left|\Delta x_{i}\right|^{p}\right]^{\frac{1}{p}} .
\end{aligned}
$$

We can use this formula when the precision of the inputs are known. For example if we know that the absolute value of the change is less than $\varepsilon$ for all $i$ $\left(\left|\Delta x_{i}\right|<\varepsilon\right)$ then we have

$$
\begin{aligned}
|\Delta M| & \leq\left[\sum_{i=1}^{n}\left(w_{i}^{1 / p} \varepsilon\right)^{p}\right]^{\frac{1}{p}}=\left[\sum_{i=1}^{n} w_{i} \varepsilon^{p}\right]^{\frac{1}{p}}, \\
& =\varepsilon \cdot\left[\sum_{i=1}^{n} w_{i}\right]^{\frac{1}{p}}=\varepsilon,
\end{aligned}
$$

so in this case the output value is less than $\varepsilon$ also. Another way when we give upper bounds with the norm of the change of the input vector. Based on the previous upper estimation we get that

$$
\begin{aligned}
|\Delta M| & \leq\left[\sum_{i=1}^{n} w_{i} \cdot\left|\Delta x_{i}\right|^{p}\right]^{\frac{1}{p}}, \\
& \leq\left[\sum_{i=1}^{n}\left(\max \left(w_{i}\right)^{1 / p}\left|\Delta x_{i}\right|\right)^{p}\right]^{\frac{1}{p}}, \\
& =\max \left(w_{i}\right)^{1 / p} \cdot\|\Delta \underline{x}\|_{p}, \\
& =\left\|\underline{w}^{1 / p}\right\|_{\infty} \cdot\|\Delta \underline{x}\|_{p} .
\end{aligned}
$$

We note that in this case some information is lost because only the norm of the change is used, but not the whole vector. As in the previous example if we know that the absolute value of the change is less then $\varepsilon$ for all $i\left(\left|\Delta x_{i}\right|<\varepsilon\right)$, now we get weaker estimation:

$$
|\Delta M| \leq\left[\sum_{i=1}^{n} \varepsilon^{p}\right]^{\frac{1}{p}}=\left[n \cdot \varepsilon^{p}\right]^{\frac{1}{p}}=n^{1 / p} \cdot \varepsilon .
$$

If the parameter of the aggregation operator is $p$, but we would like to measure the change of the input vector in $q$ norm, then we have to switch form $p$ to $q$ using the properties of $p$-norm. We handle the two kind of upper estimations on $|\Delta M|$ as different cases. then

If the starting point is that $|\Delta M| \leq\left\|\underline{w}^{1 / p} \cdot \Delta \underline{x}\right\|_{p}$

- if $p \leq q$ then

$$
\begin{aligned}
|\Delta M| & \leq\left\|\underline{w}^{1 / p} \cdot \Delta \underline{x}\right\|_{p} \leq n^{1 / p-1 / q} \cdot\left\|\underline{w}^{1 / p} \cdot \Delta \underline{x}\right\|_{q}, \\
& \leq n^{1 / p-1 / q} \cdot\left\|\underline{w}^{1 / p}\right\|_{\infty} \cdot\|\Delta \underline{x}\|_{q} \cdot
\end{aligned}
$$

- if $p>q$ then

$$
\begin{aligned}
|\Delta M| & \leq\left\|\underline{w}^{1 / p} \cdot \Delta \underline{x}\right\|_{p} \leq\left\|\underline{w}^{1 / p} \cdot \Delta \underline{x}\right\|_{q}, \\
& \leq\left\|\underline{w}^{1 / p}\right\|_{q} \cdot \| \Delta \underline{x}_{q} .
\end{aligned}
$$

If use the estimation $|\Delta M| \leq \max \left(w_{i}\right)^{1 / p} \cdot\|\underline{x} \underline{x}\|_{p}$ then

- if $p \leq q$ then

$$
\begin{aligned}
|\Delta M| & \leq \max \left(w_{i}\right)^{1 / p} \cdot\|\Delta \underline{x}\|_{p}, \\
& \leq \max \left(w_{i}\right)^{1 / p} \cdot n^{1 / p-1 / q} \cdot\|\Delta \underline{x}\|_{q}, \\
& =\left\|\underline{w}^{1 / p}\right\|_{\infty} \cdot n^{1 / p-1 / q} \cdot\|\underline{x}\|_{q} .
\end{aligned}
$$

- if $p>q$ then

$$
\begin{aligned}
|\Delta M| & \leq \max \left(w_{i}\right)^{1 / p} \cdot\|\Delta \underline{x}\|_{p}, \\
& \leq \max \left(w_{i}\right)^{1 / p} \cdot\|\Delta \underline{x}\|_{q}, \\
& =\left\|\underline{w}^{1 / p}\right\|_{\infty} \cdot\|\Delta \underline{x}\|_{q} .
\end{aligned}
$$

It is easy to check that the bounds from the second estimation are weaker.

\subsection{Special case: equal weights}

A special case worth mentioning is when $w_{i}=$ $1 / n$ for all $i$. The computations and the final formu- 
las are much more simpler than in general case.

$$
\begin{aligned}
|\Delta M| & =\left|\left[\sum_{i=1}^{n} \frac{1}{n} x_{i}^{* p}\right]^{\frac{1}{p}}-\left[\sum_{i=1}^{n} \frac{1}{n} x_{i}^{p}\right]^{\frac{1}{p}}\right| \\
& =\left(\frac{1}{n}\right)^{1 / p} \cdot\left|\left[\sum_{i=1}^{n} x_{i}^{* p}\right]^{\frac{1}{p}}-\left[\sum_{i=1}^{n} x_{i}^{p}\right]^{\frac{1}{p}}\right| \\
& =\left(\frac{1}{n}\right)^{1 / p} \cdot\left|\left\|\underline{x}^{*}\right\|_{p}-\|\underline{x}\|_{p}\right| \\
& \leq\left(\frac{1}{n}\right)^{1 / p} \cdot\|\Delta \underline{x}\|_{p} .
\end{aligned}
$$

If the change of the input vector is measured in other norm $(q)$ then

- if $p \leq q$ then

$$
\begin{aligned}
|\Delta M| & \leq\left(\frac{1}{n}\right)^{1 / p} \cdot\|\Delta \underline{x}\|_{p}, \\
& \leq\left(\frac{1}{n}\right)^{1 / p} \cdot n^{1 / p-1 / q} \cdot\|\Delta \underline{x}\|_{q}, \\
& =n^{-1 / q} \cdot\|\Delta \underline{x}\|_{q} .
\end{aligned}
$$

- if $p>q$ then

$$
|\Delta M| \leq\left(\frac{1}{n}\right)^{1 / p} \cdot\|\Delta \underline{x}\|_{p} \leq n^{-1 / p} \cdot\|\Delta \underline{x}\|_{q} .
$$

So the general form is

$$
|\Delta M| \leq n^{-\min (1 / p, 1 / q)} \cdot\|\Delta \underline{x}\|_{q}
$$

\section{Sensitivity of a Fuzzy Signature}

\subsection{General case}

Applying the results of the previous Section we can analyse the sensitivity of fuzzy signatures in which the values are determined by a WGM operator in every nodes. The sensitivity bound of the whole fuzzy signature can be derived from the bounds of the WGM-s, according to the graph structure of the signature. The whole computation can be carried out from the leaves of the signature to the root.

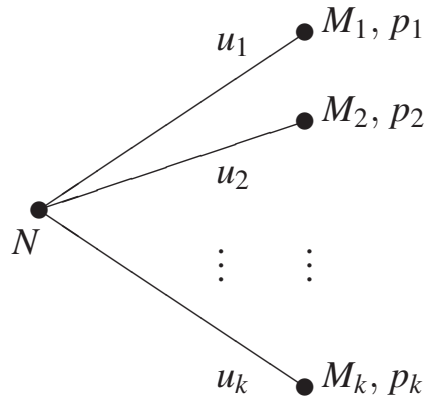

Figure 2. A part of a fuzzy signature.

Let us denote the inputs of $M_{i}$ by $x_{i j}, j=$ $1, \ldots, n_{i}$, and the weights of the inputs by $w_{i j}, j=$ $1, \ldots, n_{i}$, so we have

$$
M_{i}=\left[\sum_{j=1}^{n_{i}} w_{i j} \cdot x_{i j}^{p_{i}}\right]^{1 / p_{i}}
$$

Then upper estimation of the change of $M_{i}$ is given by the previous Section:

$$
\left|\Delta M_{i}\right| \leq\left[\sum_{j=1}^{n_{i}} w_{i j} \cdot \Delta x_{i j}^{p_{i}}\right]^{1 / p_{i}}=\left\|\underline{w i}^{1 / p_{i}} \cdot \Delta \underline{x_{i}}\right\|_{p_{i}}
$$

Let us denote the minimum of $p_{1}, p_{2}, \ldots, p_{k}$ by $p_{*}$. Then, because of the properties of the $p$-norm the following holds for any $i=1,2, \ldots, k$ :

$$
\left\|\underline{w}_{i}^{1 / p_{i}} \cdot \Delta \underline{x}_{i}\right\|_{p_{i}} \leq\left\|\underline{w}_{i}^{1 / p_{i}} \cdot \Delta \underline{\underline{x}}\right\|_{p_{*}} .
$$

Moreover

$$
\left\|\underline{w}_{i}^{1 / p_{i}} \cdot \Delta \underline{x}_{i}\right\|_{p_{*}} \leq\left\|\underline{w i}^{1 / p_{i}}\right\|_{p_{*}} \cdot\left\|\underline{\Delta} \underline{x}_{i}\right\|_{p_{*}} .
$$

Using the above upper estimations we get an upper estimation for the change of the next stage ( $N$, see Figure 2), where

$$
\begin{aligned}
\underline{u}^{1 / q} & =\left(u_{1}^{1 / q}, \ldots, u_{k}^{1 / q}\right), \\
\Delta \underline{M} & =\left(\Delta M_{1}, \ldots, \Delta M_{k}\right), \\
\underline{u}^{1 / q} \cdot \Delta \underline{M} & =\left(u_{1}^{1 / q} \cdot \Delta M_{1}, \ldots, u_{k}^{1 / q} \cdot \Delta M_{k}\right) .
\end{aligned}
$$


The upper bound is

$$
\begin{aligned}
|\Delta N| & \leq\left\|\underline{u}^{1 / q} \cdot \underline{\Delta M}\right\|_{q}=\left[\sum_{i=1}^{k} u_{i} \cdot\left|\Delta M_{i}\right|^{q}\right]^{1 / q} \\
& \leq\left[\sum_{i=1}^{k} u_{i} \cdot\left\|\underline{w}_{i}^{1 / p_{i}} \cdot \Delta \underline{x}_{i}\right\|_{p_{i}}^{q}\right]^{1 / q} \\
& \leq\left[\sum_{i=1}^{k} u_{i} \cdot\left\|\underline{w}_{i}^{1 / p_{i}} \cdot \Delta \underline{x}_{i}\right\|_{p_{*}}^{q}\right]^{1 / q} \\
& =\left[\sum_{i=1}^{k}\left(u_{i}^{1 / q} \cdot\left\|\underline{w}_{i}^{1 / p_{i}} \cdot \Delta \underline{x}_{i}\right\|_{p_{*}}\right)^{q}\right]^{1 / q} \\
& =\left\|\underline{u}^{1 / q} \cdot\right\| \underline{w}^{1 / p_{i}} \cdot \Delta \underline{x}_{p_{*}} \|_{q} \cdot
\end{aligned}
$$

Here the last term is the $q$-norm of a vector whose $i$ th element is

$$
u_{i}^{1 / q} \cdot\left[\sum_{j=1}^{n_{i}}\left(w_{i j}^{1 / p_{i}} \cdot \Delta x_{i j}\right)^{p_{*}}\right]^{1 / p_{*}} .
$$

As we can see, in general case a closed, elegant formula couldn't be given, but only recursive method which can be applied from level to level.

\subsection{Special cases}

The sensitivity and complexity of a fuzzy signature mostly depend on the structure of the graph and on the aggregation operators applied in the nodes. According to this fact several special cases can be distinguished. In this Section we examine the case when the fuzzy signature is equipped with very similar aggregation operators and the case when the graph is a (maybe not perfect) full $n$-ary graph.

\subsubsection{Homogeneous fuzzy signatures}

The sensitivity analysis of a fuzzy signature becomes much more simple if the value of the parameter $p$ is the same for all of the WGM operators applied in the nodes. If this condition holds, the output vale of the signature is the weighted generalized mean of the input values with parameter $p$, where the weights are the product of the weights form the root to the leaves.

Definition 4 A fuzzy signature is called homogeneous if all of the aggregation operators in the nodes are weighted generalized mean operators with the same value of $p$.
Lemma 2 The $W G M$ of $y_{1}, \ldots, y_{k}$ with weights $v_{1}, \ldots, v_{k}$ and with parameter $p$ where all of the $y_{i^{-}}$ $s$ are WGM's of $x_{j i}$-s with weights $w_{1 i}, \ldots, w_{n_{i}}$ and with the same parameter of $p$, is the WGM of the $x$-s with weights $v_{i} \cdot w_{j i}$

Proof:

$$
\begin{aligned}
& {\left[\sum_{i=1}^{k} v_{i} \cdot y_{i}^{p}\right]^{\frac{1}{p}}=\left[\sum_{i=1}^{k} v_{i} \cdot\left[\left[\sum_{j=1}^{n_{i}} w_{j i} \cdot x_{j i}^{p}\right]^{\frac{1}{p}}\right]^{p}\right]^{\frac{1}{p}}} \\
& =\left[\sum_{i=1}^{k} \sum_{j=1}^{n_{i}} v_{i} \cdot w_{j i} \cdot x_{j i}^{p}\right]^{\frac{1}{p}}=\left[\sum_{l=1}^{\sum n_{i}} c_{l} \cdot x_{l}^{p}\right]^{\frac{1}{p}} \cdot
\end{aligned}
$$

So the sensitivity analysis of a homogeneous fuzzy signature is nothing else but the simple sensitivity analysis of only one weighted generalized mean aggregation operator, which was discussed in details in Section 3.

\subsubsection{Full $n$-ary fuzzy signatures with equal weights}

The $n$-ary fuzzy signature is a fuzzy signature which has an $n$-ary tree graph representation. An $n$-ary tree is a tree graph in which each node has no more than $n$ children. A full $n$-ary tree is an $n$-ary tree where within each level every node has either 0 or $n$ children; a perfect $n$-ary tree is a full $n$-ary tree in which all leaf nodes are at the same depth (the distance from the leaf to the root is the same).

We consider the case when the weights are equal, so for a full $n$-ary fuzzy signature the weights are $1 / n$ for all of the nodes.

If the signature is homogeneous, then (according to the previous subsection) it can be transformed into one simple weighted generalized mean of the inputs. For example, if we think of the tree graphs from Figure 3 as homogeneous fuzzy signatures, then the weights on 1.h.s. are $1 / 9$ for all of the inputs, and $1 / 3,1 / 9, \ldots, 1 / 9$ on r.h.s.

In general, a perfect homogeneous $n$-ary fuzzy signature with $k$ levels is equivalent to a weighted general mean where the weights are $1 / n^{k}$ for all of the inputs. Then the upper bound of the change of 
this WGM is following:

$$
|\Delta M| \leq\left(\frac{1}{n}\right)^{k / p} \cdot\|\Delta \underline{x}\|_{p} .
$$

If the tree graph is not perfect (but still full) then an input value $x_{i}$ has the weight $1 / n^{l_{i}}$, where $l_{i}$ denotes the level of $x_{i}$, the number of inputs is $m$. Then the upper bound of the change of the corresponding WGM is

$$
\begin{aligned}
|\Delta M| & \leq\left[\sum_{i=1}^{m}\left(\frac{1}{n}\right)^{l_{i}} \cdot\left|\Delta x_{i}\right|^{p}\right]^{1 / p} \\
& \leq\left(\frac{1}{n}\right)^{l_{*} / p} \cdot\|\Delta \underline{x}\|_{p}
\end{aligned}
$$

where $l_{*}$ denotes the minimum of the levels.
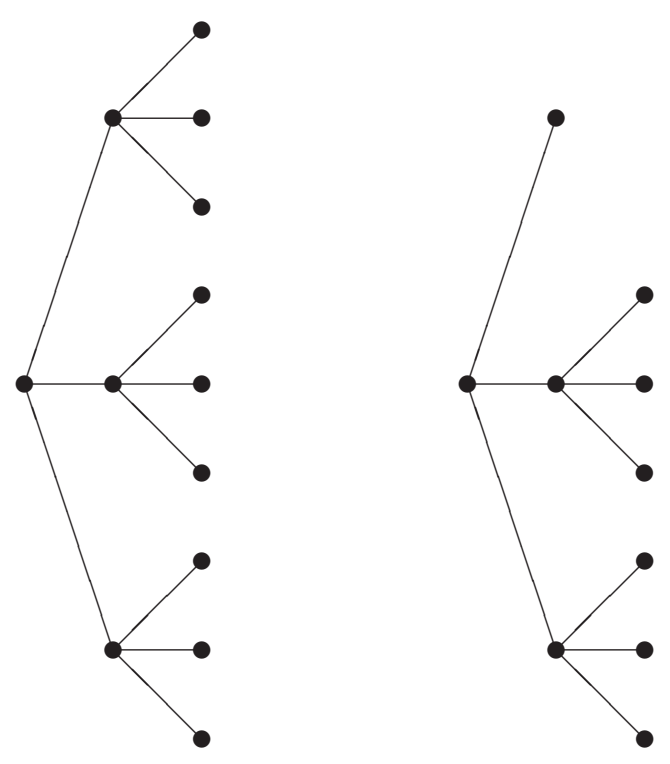

Figure 3. A perfect ternary fuzzy signature graph (left), and a not perfect ternary fuzzy signature graph (right).

If the fuzzy signature is not homogeneous, then the computation can be carried out as in 4.1, but the weights are everywhere $1 / n$ :

$$
\begin{aligned}
|\Delta N| & \leq\left[\sum_{i=1}^{n} \frac{1}{n} \cdot\left|\Delta M_{i}\right|^{q}\right]^{1 / q} \\
& \leq\left\|\underline{1 / n^{1 / q}} \cdot\right\| \underline{1 / n^{1 / p_{i}}} \cdot \Delta \underline{x}\left\|_{p_{*}}\right\|_{q},
\end{aligned}
$$

where the last term is the $q$-norm of a vector whose $i$ th element is

$$
\left(\frac{1}{n}\right)^{1 / q} \cdot\left[\sum_{j=1}^{n}\left(\left(\frac{1}{n}\right)^{1 / p_{i}} \cdot \Delta x_{i j}\right)^{p_{*}}\right]^{1 / p_{*}},
$$

and $p_{*}$ denotes the minimum of $p_{1}, \ldots, p_{n}$.

\section{Example From Civil Engineering}

In this Section we discuss the sensitivity of a fuzzy signature which was applied for statusdetermining and ranking buildings of similar age and structural arrangement.

In Budapest city a lot of old residential buildings are available of similar age and structural arrangement. At the end of the 19th and at the beginning of the 20th centuries the number of inhabitants increased from 280000 to 730000 . In this time period new city districts were constructed with the application of the technological methods, which were known at that time. A significant part of these residential buildings still constitutes the dominant element of the current townscape. It is one of the most pressing issues of the Hungarian capital that a considerable part of these buildings are in degraded condition. The modernization and renovation of these buildings and their ranking from the aspect of the urgency of their renovation are significant task due to the limited financial possibilities.

A decision-supporting model was created by applying the fuzzy signature [15]. This model is suitable for the ranking and qualification of residential buildings. The model was used for the first time on a database, which is based on expert opinions. After that a tree-structure, necessary for the examination of the load-bearing structures of buildings, were prepared. Primary structures (main loadbearing structures) and secondary structures (so not main load-bearing structures which play an important role in the protection of the main load bearing structures) were differentiate during the research, in this article we deal only with the branch of the 
primary structures. With the help of this branch it is possible to make a ranking of the load bearing structures of the examined buildings based on their arrangement, materials and conditions. The examined load bearing structures used in the model are as follows: foundation structures, wall structures, floor structures, side corridor structures, step structures and roof structures. The database was prepared on the basis of the research of more than hundred buildings, typical in Budapest, so the results achieved, well reflect the actual conditions of this type of residential buildings.

The structure of the signature is shown in Figure 4. The names and meanings of the input and internal variables are listed below.

The input variables:

$x_{1}$ : foundation structures

$x_{2}$ : wall structures

$x_{3}$ : cellar floor

$x_{4}:$ intermediate floor

$x_{5}$ : cover floor

$x_{6}:$ side corridor structures

$x_{7}:$ step structures

$x_{8}$ : facade

$x_{9}$ : footing

$x_{10}:$ roof structures

$x_{11}$ : roof covering

$x_{12}:$ tin structures

$x_{13}$ : insulation against soil moisture and ground water

The internal variables:

$h_{1}$ : floor structures

$h_{2}$ : vertical load-bearing structures

$h_{3}$ : horisontal load-bearing structures

$h_{4}:$ primary structures

$h_{5}:$ surface formation

$h_{6}:$ secondary structures

$h_{7}:$ primary and secondary structures
This is a homogeneous fuzzy signatures with parameter $p=1$ and with the following weights:

$$
\begin{aligned}
w_{1,1} & =0.75, & w_{1,2} & =0.25, \\
w_{2,1} & =0.4, & w_{2,2} & =\frac{0.6 \cdot n}{n+1}, \\
w_{2,3} & =\frac{0.6}{n+1}, & w_{2,4} & =\frac{0.4}{0.8+0.2 \cdot n}, \\
w_{2,5} & =\frac{0.2 \cdot n}{0.8+0.2 \cdot n}, & w_{2,6} & =\frac{0.2}{0.8+0.2 \cdot n}, \\
w_{2,7} & =\frac{0.2}{0.8+0.2 \cdot n}, & w_{3,1} & =0.55-0.05 \cdot n, \\
w_{3,2} & =0.45+0.05 \cdot n, & w_{3,3} & =\frac{0.65}{0.8+0.2 \cdot f}, \\
w_{3,4} & =\frac{0.2 \cdot f}{0.8+0.2 \cdot f}, & w_{3,5} & =\frac{0.15}{0.8+0.2 \cdot f}, \\
w_{3,6} & =1-\frac{0.5}{n}, & w_{3,7} & =\frac{0.5}{n},
\end{aligned}
$$

$$
\begin{aligned}
w_{4,1} & =\frac{0.35 \cdot m}{0.2+0.45 \cdot(n-1)+0.35 \cdot m}, \\
w_{4,2} & =\frac{0.45 \cdot(n-1)}{0.2+0.45 \cdot(n-1)+0.35 \cdot m}, \\
w_{4,3} & =\frac{0.2}{0.2+0.45 \cdot(n-1)+0.35 \cdot m} .
\end{aligned}
$$

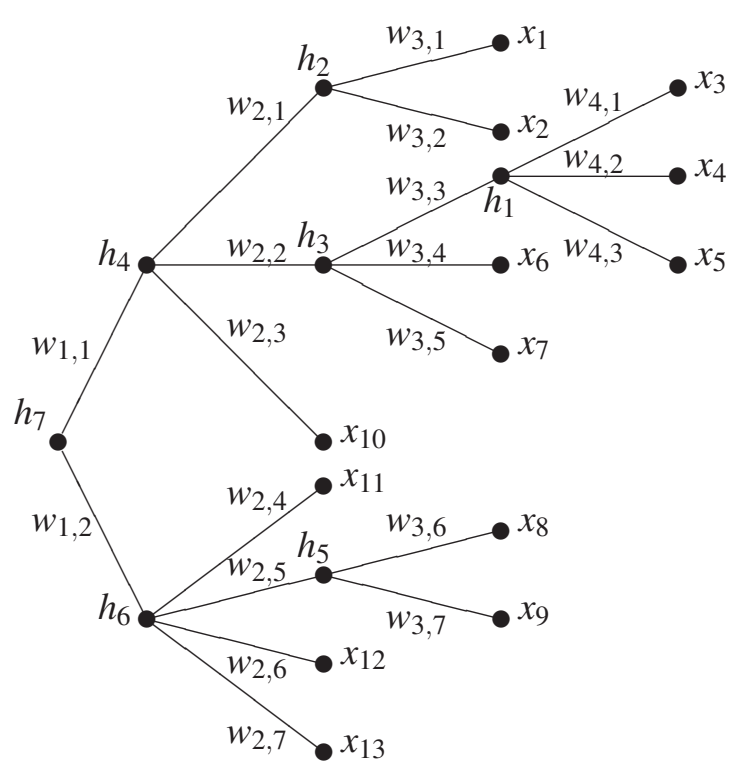

Figure 4. A fuzzy signature for status-determining and ranking buildings. 
The possible values of the parameters:

- $n=2,3,4,5$ (number of the storeys of the building),

$-0 \leq m \leq 1$ (extend of the cellar built),

- $f=0$ or 1 (building with or without side corridor).

The input values $\left(x_{i}\right.$-s) are real numbers between 0 and 1 according to the opinion of a human expert about the status of the $i$-th partial structure. The final output is the membership value of $h_{7}$. If a building is surveyed by different experts, then their opinion about the status of partial structures may result in different values of $h_{7}$. The following question arises: if there are small differences between the ratings given by the experts to the partial structures, then how large can be the deviation between the final scores of a building? In other words, how sensitive is this fuzzy signature to small perturbations?

This is a homogeneous fuzzy signature, so we can analyse it as a simple WGM. From the results of Section 4 it follows that

$$
\left|\Delta h_{7}\right| \leq \sum_{i=1}^{13} v_{i} \cdot\left|\Delta x_{i}\right|
$$

where $v_{i}$ is the weight of the $i$ th input, computed as the product of the weights from the root to the $i$ th leaf (for example with the notations of Figure 4 $\left.v_{1}=w_{1,1} \cdot w_{2,1} \cdot w_{3,1}\right)$. If we measure the sensitivity in one of the well-known vector norms of the change, then

$$
\begin{aligned}
& \left|\Delta h_{7}\right| \leq \max \left(v_{i}\right) \cdot\|\Delta \underline{x}\|_{1}, \\
& \left|\Delta h_{7}\right| \leq \sqrt{13} \cdot \max \left(v_{i}\right) \cdot\|\Delta \underline{x}\|_{2}, \\
& \left|\Delta h_{7}\right| \leq 13 \cdot \Delta\|\underline{x}\|_{\infty} .
\end{aligned}
$$

Actual values of the $v_{i}$-s depend on the actual values of the parameters $(m, n, f)$, as it is shown in Table 1.

We can conclude that this signature is not too sensitive, namely a small change in the partial opinions do not yields a large difference between the final conclusions. If the absolute values of the differences of the ratings given by the human experts are than $\varepsilon$ for all of the variables, then the difference between the final conclusions are less $\varepsilon$, too.

Table 1. Examples for the weights of the input variables for different values of the parameters (rounded to four decimals).

\begin{tabular}{|c||c|c|}
\hline & $\begin{array}{c}n=3, f=1, \\
m=1\end{array}$ & $\begin{array}{c}n=4, f=0, \\
m=0.5\end{array}$ \\
\hline \hline$x_{1}$ & 0.1200 & 0.1050 \\
$x_{2}$ & 0.1800 & 0.1950 \\
$x_{3}$ & 0.0530 & 0.0297 \\
$x_{4}$ & 0.1362 & 0.2289 \\
$x_{5}$ & 0.0303 & 0.0339 \\
$x_{6}$ & 0.0675 & 0.0000 \\
$x_{7}$ & 0.0506 & 0.0675 \\
$x_{8}$ & 0.1125 & 0.0900 \\
$x_{9}$ & 0.0714 & 0.0625 \\
$x_{10}$ & 0.0893 & 0.1094 \\
$x_{11}$ & 0.0179 & 0.0156 \\
$x_{12}$ & 0.0357 & 0.0312 \\
$x_{13}$ & 0.0357 & 0.0312 \\
\hline
\end{tabular}

\section{Conclusions}

The sensitivity of the weighted generalized mean aggregation operator for parameter value $p \geq$ 1 was discussed via Minkowski's inequality and in terms of various vector norms of the input vector. Applying these results the sensitivity of fuzzy signatures equipped with WGMs was also discussed. In general case a recursive estimation can be given, but in the special case when the WGMs have the same parameter, the sensitivity analyses of a fuzzy signature simplified to a sensitivity analyses of a single WGM. The complexity of the fuzzy signature is influenced by the structure and the aggregation operators also, but the aggregation operators play the main role: complexity can be highly reduced if we use the same kind of aggregation operators.

A real-life example from civil engineering was also discussed, we analysed the sensitivity of a method for status-determining and ranking buildings. It was concluded that the applied fuzzy signature is not too sensitive: if the opinions of the experts about the partial structures are relatively close to each other, then the final evaluations of the whole building will be close to each other, too. 


\section{Acknowledgement}

This work was supported by the Hungarian Scientific Research Fund (OTKA) K105529 and K108405.

\section{References}

[1] K. W. Wong, T. D. Gedeon, L. T. Kóczy, Construction of fuzzy signature from data: an example of SARS pre-clinical diagnosis system, in: Proceedings of the IEEE International Conference on Fuzzy Systems (FUZZ-IEEE2004), Budapest, Hungary, 2004, pp.1649-1654.

[2] Á. Ballagi, L. T. Kóczy, T. D. Gedeon, Robot cooperation without explicit communication by fuzzy signatures and decision trees, in: Proceedings of theJoint 2009 International Fuzzy Systems Association World Congress and 2009 European Society of Fuzzy Logic and Technology Conference (IFSA-EUSFLAT2009), Lisbon, Portugal, 2009, pp.1468-1473.

[3] T. Vámos, L. T. Kóczy , G. Biró, Fuzzy signatures in datamining, in: Proceedings of the Joint 9th IFSA World Congress and 20th NAFIPS International Conference, Vancouver, BC, Canada, 2001, pp. 2842-2846 (5).

[4] G. Molnárka, L. T. Kóczy, Decision Support System for Evaluating Existing Apartment Buildings Based on Fuzzy Signatures, Int. J. of Computers , Communications \& Control, 2011, No. 3, pp. 442457.

[5] C. Pozna, N. Minculete, R. E. Precup, L. T. Kóczy, Á. Ballagi, Signatures: Definitions, operators and applications to fuzzy modelling, Fuzzy Sets and Systems 201 (2012), pp. 86-104.

[6] L. T. Kóczy, T. Vámos, G. Biró, Fuzzy signatures, in: Proceedings of the 4th Meeting of the Euro

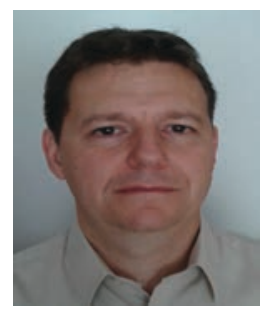

Istvan A. Harmati received his MSc from Eotvos Lorand University in 2001, in mathematics and physics. He received his $\mathrm{PhD}$ degree in mechanical engineering from the Technical University of Budapest in 2009, habilitation from Szechenyi Istvan University in 2015. He is an associate professor at the Department of Mathematics and Computational Sciences, at Szechenyi Istvan University. His main research interests are mathematical modelling and analysis of uncertain systems, including stochastic (probabilistic)
Working Group on Fuzzy Sets and the 2nd International Conference on Soft and Intelligent Computing (EUROPUSE-SIC99), Budapest, Hungary, 1999, pp. 210-217.

[7] J.A.Goguen, L-fuzzy sets, J. Math. Anal. Appl. 18(1) (1967), pp. 145-174.

[8] I. Á. Harmati, Á. Bukovics, L. T. Kóczy, Sensitivity Analysis of the Weighted Generalized Mean Aggregation Operator and its Application to Fuzzy Signatures, IEEE World Congress on Computational Intelligence (WCCI 2014 - FUZZ-IEEE 2014). Peking, Kna, 2014.07.06-2014.07.11. New York: IEEE, 2014. pp. 1327-1332.

[9] U. Kaymak, H.R. van Nauta Lemke, T. Boer: A sensitivity-based analysis of weighted fuzzy aggregation, In: Proceedings of the IEEE World Congress on Computational Intelligence, IEEE International Conference on Fuzzy Systems, IEEE, 1998. pp. 755-760.

[10] V. Torra: Sensitivity analysis for WOWA, OWA and WM operators, In: Proceedings of ISIE 2001, IEEE International Symposium on Industrial Electronics, IEEE, 2001. pp. 134-137.

[11] M. Zarghami, F. Szidarovszky: Fuzzy quantifiers in sensitivity analysis of OWA operator, Computers \& Industrial Engineering 54 (2008), pp. 10061018.

[12] G. H. Hardy, J. E. Littlewood, G. Pólya: Inequalities, Cambridge University Press, 1952.

[13] P. S. Bullen: Handbook of means and their inequalities, Kluwer Academic Publishers, 2003.

[14] G. H. Golub, C. F. van Loane: Matrix computations, John Hopkins University Press, 1996.

[15] Á. Bukovics, L.T. Kóczy, Fuzzy Signature-based Model for Qualification and Ranking of Residential Buildings, XXXVIII. IAHS World Congress on Housing, Istanbul, Turkey, 2012. pp. 290-297

and fuzzy point of view, fuzzy mathematics, probability and possibility theory and stochastic processes.

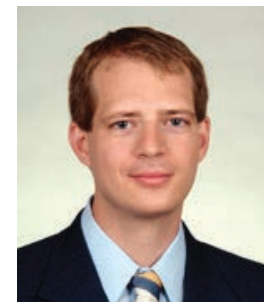

Adam Bukovics's degree earned: Technical University of Budapest: MSc in Civil Engineering. He received his PhD at the Széchenyi István University, Győr, Hungary. Research interest: building diagnostics, building pathology, fuzzy signatures. Membership in scientific societies: CIB W086 Building Pathology Commission. 


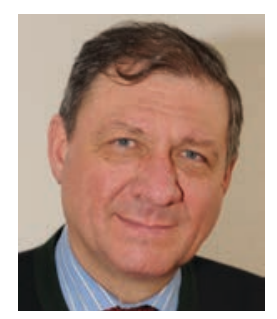

Laszlo T. Koczy received the MSc, MPhil and PhD degrees from the Technical University of Budapest in 1975, 1976 and 1977, respectively; and the DSc degree from the Hungarian Academy of Science in 1998. He spent his career at BME until 2001, and from 2002 at Szechenyi Istvan University. He has been from 2002 to 2011 Dean of Engineering, and from 2013 to current President of the University Research Council and of the University PhD Council. From 2012 he has been a member of the Hungarian Accreditation Committee, elected Chair of the Engineering and Computer Science sub-committee, member of the Professors and $\mathrm{PhD}$ sub-committee, and has been a member of the National Doctoral Council since 2012.

His research interests are fuzzy systems, evolutionary algorithms and neural networks as well as applications. 